\title{
Left Cardiac Artery Dominance
}

National Cancer Institute

\section{Source}

National Cancer Institute. Left Cardiac Artery Dominance. NCI Thesaurus. Code C99971.

The posterior descending artery (PDA) and posterolateral artery (PLA) arises from the left circumflex artery. (ACC) 